\title{
Paternal physical exercise improves spatial learning ability by enhancing hippocampal neuroplasticity in male pups born from obese maternal rats
}

\author{
Hye-Sang Park, Tae-Woon Kim* \\ Department of Physiology, College of Medicine, Kyung Hee University, Seoul, Korea
}

Maternal obesity exerts negative effects on cognitive function and behavior of the offspring. In the present study, we assessed the effects of paternal physical exercise on spatial learning ability in relation with hippocampal neuroplasticity in the rat pups born from the obese maternal rats. There were four experimental groups: paternal nonexercised male pups from normal maternal rats, paternal exercised male pups from normal maternal rats, paternal nonexercised male pups from obese maternal rats, and paternal exercised male pups from obese maternal rats. Normal diet was supplied for normal maternal rats and high-fat diet was supplied for obese maternal rats for a 12-week period until mating, and the same diet for each group continued throughout pregnancy and lactation period. Male rats in the exercising groups exercised for a 12 week period. Spatial learning ability was reduced in the male rat pups born from the obese maternal rats. Expressions of brain-derived neurotrophic factor (BDNF) and tyrosine kinase B receptor (TrkB) in the hippocampus were suppressed and cell proliferation and differentiation in the hippocampus were reduced in the male rat pups born from the obese maternal rats. Paternal treadmill exercise improved spatial learning ability, increased BDNF and TrkB expressions, and enhanced cell proliferation and differentiation in the male rat pups born from the obese maternal rats. It can be suggested that paternal exercise enhances hippocampal neuroplasticity and consequently improved spatial learning ability in the rat pups born from the obese maternal rats.

Keywords: Paternal exercise, Maternal obesity, Offspring, Spatial learning ability, Hippocampus

\section{INTRODUCTION}

Obesity is a risk factor not only for the cardiovascular diseases but also for the brain-related disorders (Elias et al., 2005; Wolf et al., 2007). Nutritional supply in the early stage after birth depends on the maternal conditions and excessive consumption of certain nutrients by mothers exerts detrimental effect on the brain functions of offspring (Férézou-Viala et al., 2007; Tozuka et al., 2009a; Tozuka et al., 2010). Nutrients, hormones, and growth factors are transported from the mother to the offspring through the placenta or breastfeeding (Kodomari et al., 2009; Raiten et al., 2007). Maternal obesity exerts negative effects on cognitive function and behavior of offspring (Tozuka et al., 2009a, 2009b).

Hippocampus is a key part of the brain involved in the process- es of learning process and memory formation. Active neurogenesis occurs in the hippocampus after birth and hippocampus is the brain area with greatest neuroplasticity (Fuchs and Gould, 2000; Lie et al., 2004).

Brain-derived neurotrophic factor (BDNF) has emerged as a key regulator of activity-dependent synaptic plasticity, and BDNF plays a key role in spatial learning ability (Donovan et al., 2008). BDNF controls development, survival, and differentiation of the neurons through the tyrosine kinase B receptor (TrkB) (Bramham and Messaoudi, 2005). 5-Bromo-2'-deoxyuridine (BrdU) is a synthetic nucleoside that is an analog of thymidine. BrdU is commonly used in the detection of proliferating cells in living tissues (Lehner et al., 2011). Doublecortin (DCX) is a marker for the cellular differentiation and DCX is associated with structural plas-

\footnotetext{
${ }^{*}$ Corresponding author: Tae-Woon Kim (i) http://orcid.org/0000-0001-8832-0874 Department of Physiology, College of Medicine, Kyung Hee University, 26 Kyungheedae-ro, Dongdaemun-gu, Seoul 02447, Korea Tel: +82-2-961-0282, Fax: +82-2-964-2195, E-mail: twkim0806@naver.com Received: May 19, 2017 / Accepted: June 5, 2017
}

This is an Open Access article distributed under the terms of the Creative Commons Attribution Non-Commercial License (http://creativecommons.org/licenses/by-nc/4.0/) which permits unrestricted non-commercial use, distribution, and reproduction in any medium, provided the original work is properly cited. 
ticity in the adult mammalian brain (Kim et al., 2013a).

Maternal exercise during pregnancy enhanced brain development of offspring by increasing neurogenesis through enhancing BDNF expression (Kim et al., 2007). Maternal exercise during pregnancy improved learning and memory in rat pups (Akhavan et al., 2008). There are many on-going studies on the relationship between maternal exercise and cognitive function of offspring.

The maternal effect is considered as an important form of the parental influence, in contrast, the paternal effect is often relatively overlooked. Half of the paternal genome is passed down to the next generation (Krawetz, 2005), therefore, the paternal conditions also should be considered as the important factor for the development of offspring. In the present study, we assessed the effects of paternal physical exercise on spatial learning ability in relation with hippocampal neuroplasticity in the rat pups born from the obese maternal rats.

\section{MATERIALS AND METHODS}

\section{Animals and treatments}

All animal experimental procedures conformed to the regulations stipulated by the National Institutes of Health and the guidelines of the Korean Academy of Medical Science. This study was approved by the Kyung Hee University Institutional Animal Care and Use Committee (KHUASP [SE]-14-018).

Male and female Sprague-Dawley rats were used (4 weeks old) for this experiment. Normal diet was supplied for normal maternal rats and high-fat diet (60\%) was supplied for obese maternal rats for a 12-week period until mating, and the same diet for each group continued throughout pregnancy and lactation period. Male rats in the exercising groups exercised for a 12-week period. Breeding conditions were maintained as a constant temperature and humidity of $20^{\circ} \mathrm{C} \pm 2{ }^{\circ} \mathrm{C}$ and $60 \%$ with a 12 -hr day and 12 hr night period.

We performed random sampling and divided the animals into following four groups $(n=5)$ : nonexercising male and normal female group, exercising male and normal female group, nonexercising male and obese female group, and exercising male and obese female group. Only male rat pups (4 weeks old) were used for the experiment. The rat pups from the above groups were redivided into following four groups $(\mathrm{n}=10)$ : paternal nonexercised male pups from normal maternal rats, paternal exercised male pups from normal maternal rats, paternal nonexercised male pups from obese maternal rats, and paternal exercised male pups from obese maternal rats.
BrdU (50 mg/kg; Sigma Chemical Co., St. Louis, MO, USA) was given intraperitoneally to all animals $1 \mathrm{hr}$ before the starting of the treadmill exercise for 3 consecutive days prior to sacrifice.

\section{Exercise protocols}

The rats in the exercise groups performed treadmill running. Exercise consisted of $5 \mathrm{~min}$ of warm up at a $0^{\circ}$ inclination at $3 \mathrm{~m} /$ $\mathrm{min}, 30 \mathrm{~min}$ of the main exercise at $10 \mathrm{~m} / \mathrm{min}$, and $5 \mathrm{~min}$ of cool down at $3 \mathrm{~m} / \mathrm{min}$ or the first 3 weeks. Following this regimen, 40 minutes of the main exercise at $10 \mathrm{~m} / \mathrm{min}$ during $4-6$ weeks, 30 min of the main exercise at $15 \mathrm{~m} / \mathrm{min}$ during 7-9 weeks, and 40 min of the main exercise at $15 \mathrm{~m} / \mathrm{min}$ during final 10-12 weeks were performed. The exercise was performed once a day and 6 days per week during 12 consecutive weeks.

\section{Morris water maze task}

Spatial learning ability was evaluated using the Morris water maze task. This task requires rats to learn the spatial location of a hidden platform in a black circular pool $(180 \mathrm{~cm}$ in diameter and $50 \mathrm{~cm}$ high) filled with clear water $\left(25^{\circ} \mathrm{C} \pm 1^{\circ} \mathrm{C}\right)$. The hidden platform $(15 \mathrm{~cm}$ in diameter and $40 \mathrm{~cm}$ high) was placed $2 \mathrm{~cm}$ below the surface of water in the middle of the north quadrant and was camouflaged by virtue of being transparent against a black background. Distal visual cues were placed on the walls around the pool. The position of the cues remained unchanged through the task. One day before training, the rat pups were habituated to swimming for $60 \mathrm{sec}$ in the pool without a platform. All rat pups were trained three times a day for four consecutive days before sacrifice.

Probe trail was conducted $24 \mathrm{hr}$ after the last training. When finding the platform, the rat pups were allowed to remain for 30 sec. If the rat pups did not find the platform within $60 \mathrm{sec}$, they were guided by hand to the platform. The rat pups were given 60 sec retention probe test, and then the platform removed from the pool. Data were automatically collected via the Smart Video Tracking System (Smart ver. 2.5, Panlab, Barcelona, Spain).

\section{Tissue preparation}

The animals were sacrificed immediately after Morris water maze task. The animals were fully anesthetized with diethyl ether, then transcardially perfused with $50-\mathrm{mM}$ phosphate-buffered saline (PBS) followed by freshly prepared $4 \%$ paraformaldehyde in 100-mM phosphate buffer ( $\mathrm{pH}, 7.4)$. The brains were then removed, postfixed in the same fixative overnight, and transferred to a $30 \%$ sucrose solution for cryoprotection. Coronal sections of 
30- $\mu \mathrm{m}$ thickness were made using a freezing microtome (Leica, Nussloch, Germany).

\section{Immunohistochemistry for BrdU}

The sections were first permeabilized by incubation in $0.5 \%$ Triton X-100 in PBS for 20 min, then pretreated in 50\% formamide- $2 \mathrm{X}$ standard saline citrate at $65^{\circ} \mathrm{C}$ for $2 \mathrm{hr}$, denatured in 2 $\mathrm{N} \mathrm{HCl}$ at $37^{\circ} \mathrm{C}$ for $30 \mathrm{~min}$, and rinsed twice in $100-\mathrm{mM}$ sodium borate $(\mathrm{pH}, 8.5)$. The sections were then incubated overnight at $4^{\circ} \mathrm{C}$ with BrdU-specific mouse monoclonal antibody (1:600; Roche, Mannheim, Germany). The sections were then washed three times with PBS and incubated with biotinylated mouse secondary antibody (1:200; Vector Laboratories, Burlingame, CA, USA) for $1 \mathrm{hr}$. The sections were then incubated for $1 \mathrm{hr}$ with an ABC complex (1:100; Vector Laboratories). For visualization, the sections were incubated in $50-\mathrm{mM}$ Tris- $\mathrm{HCl}(\mathrm{pH}, 7.6)$ containing $0.03 \%$ diaminobenzidine (DAB), $40-\mathrm{mg} / \mathrm{mL}$ nickel chloride, and $0.03 \%$ hydrogen peroxide for $5 \mathrm{~min}$.

After BrdU labeling, a mouse anti-neuronal nucleic antibody (1:1,000; Chemicon International, Temecula, CA, USA) was used on the same sections to differentiate neurons. The sections were washed 3 times with PBS, incubated for $\mathrm{l}$ hr with a biotinylated anti-mouse secondary antibody. For staining, the sections were incubated in a reaction mixture consisting of $0.03 \% \mathrm{DAB}$ and $0.03 \%$ hydrogen peroxide for $5 \mathrm{~min}$. The sections were mounted onto gelatin-coated slides, air-dried overnight at room temperature, and covers lipped under Permount (Thermo Fisher Scientific Inc., Waltham, MA, USA).

\section{Immunohistochemistry for DCX}

The sections were incubated in PBS for 10 min, washed three times in PBS, then incubated in $1 \%$ hydrogen peroxide for $30 \mathrm{~min}$. The sections were incubated overnight with goat anti-DCX antibody (1:1,000; Oncogene Research Product, Cambridge, UK). They were then incubated with the appropriate biotinylated secondary antibody (1:200; Vector Laboratories) for another $1 \mathrm{hr}$, washed, and incubated in $\mathrm{ABC}$ complex (Vector Elite $\mathrm{ABC}$ kit; 1:100; Vector Laboratories). Labeling was visualized using $0.03 \% \mathrm{DAB}$, and the sections were mounted onto gelatin-coated slides. The slides were air-dried overnight at room temperature, and the coverslips were mounted using Permount (Thermo Fisher Scientific Inc.).

\section{Western blotting for BDNF and TrkB}

Collected hippocampal samples were homogenized on ice and lysed in a lysis buffer containing $50-\mathrm{mM}$ Tris- $\mathrm{HCl}(\mathrm{pH}, 7.5), 150-$
$\mathrm{mM} \mathrm{NaCl}, 0.5 \%$ deoxycholic acid, $1 \%$ Nonidet $\mathrm{P} 40,0.1 \%$ sodium dodecyl sulfate (SDS), 1-mM phenylmethylsulfonyl fluoride, and $100-\mathrm{mg} / \mathrm{mL}$ leupeptin. Protein content was measured using a Bio-Rad colorimetric protein assay kit (Bio-Rad, Hercules, CA, USA). Thirty micrograms of total protein was separated on SDS-polyacrylamide gels and transferred onto a nitrocellulose membrane. The membrane was blocked with dehydrated milk, then incubated with mouse anti- $\beta$-actin antibody $(1: 1,000$; Santa Cruz Biotechnology, Santa Cruz, CA, USA), rabbit anti-BDNF/ TrkB antibody (1:1,000; Santa Cruz Biotechnology). After washing, horseradish peroxidase-conjugated, species appropriate secondary antibodies were applied. Incubations were performed at room temperature. The bands were detected using the enhanced chemiluminescence detection system (Santa Cruz Biotechnology), and quantified using an Image-Pro Plus computer-assisted image analysis system (Media Cyberbetics Inc., Silver Spring, MD, USA).

\section{Statistical analyses}

Cell counting and optical density quantification were performed using Image-Pro Plus (Media Cyberbetics Inc.) attached to a light microscope (Olympus, Tokyo, Japan). The data were analyzed with one-way analysis of variance, followed by Duncan post hoc tests. All values are expressed as the mean \pm standard error of the mean, and $P<0.05$ was considered significant.

\section{RESULTS}

\section{Effect of paternal treadmill exercise on spatial learning ability in male rat pups}

To assess spatial learning ability, we performed a Morris water maze task (Fig. 1). From the assessments performed on day 2, 3, and 5 , the male rat pups from the obese maternal rats showed reduced spatial learning ability compared to the male rat pups from the normal maternal rats. Paternal exercise improved spatial learning ability in the male rat pups from the obese maternal rats.

\section{Effect of paternal treadmill exercise on BDNF and TrkB expressions in male rat pups}

BDNF and TrkB expressions were quantified by western blotting (Fig. 2). Expressions of BDNF and TrkB in the hippocampus were lower in the male rat pups from the obese maternal rats compared to the male rat pups from the normal maternal rats. Paternal exercise enhanced BDNF and TrkB expressions in the male rat pups from the obese maternal rats. 

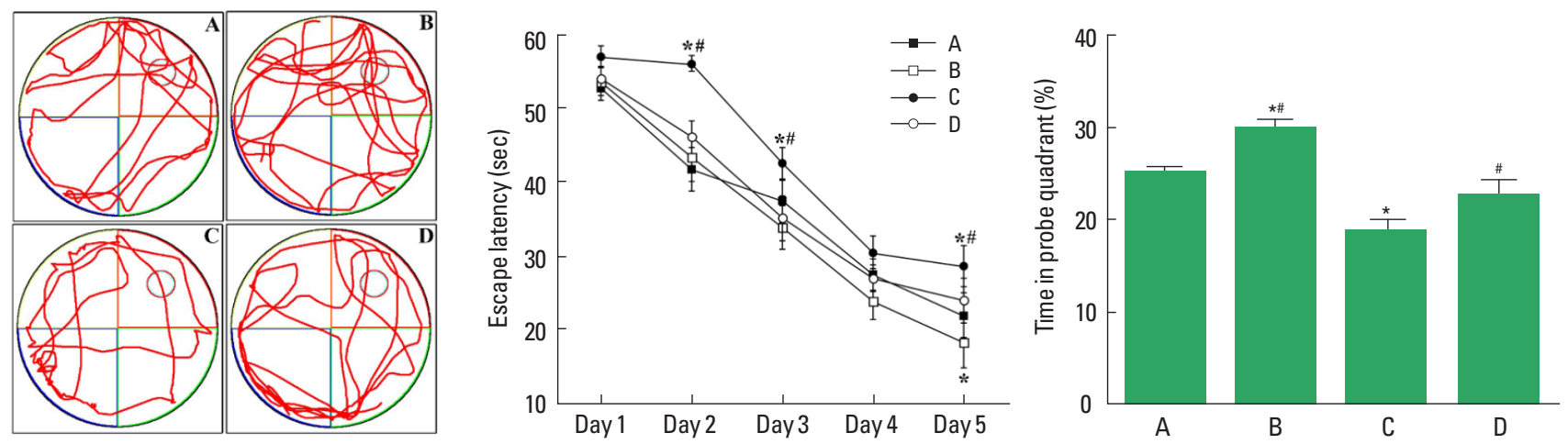

Fig. 1. Effect of paternal treadmill exercise on spatial learning ability in male rat pups. Left panel: Swimming pathway. Middle panel: Daily escape latency in Morris water maze task. Right panel: Time in probe quadrant in Morris water maze task. A, paternal nonexercised male pups from normal maternal rats; B, paternal exercised male pups from normal maternal rats; $C$, paternal nonexercised male pups from obese maternal rats; $D$, paternal exercised male pups from obese maternal rats. Data are expressed as the mean \pm standard error of the mean. ${ }^{*} P<0.05$ compared to the paternal nonexercised male pups from normal maternal rats. ${ }^{\#} P<0.05$ compared to the paternal nonexercised male pups from obese maternal rats.
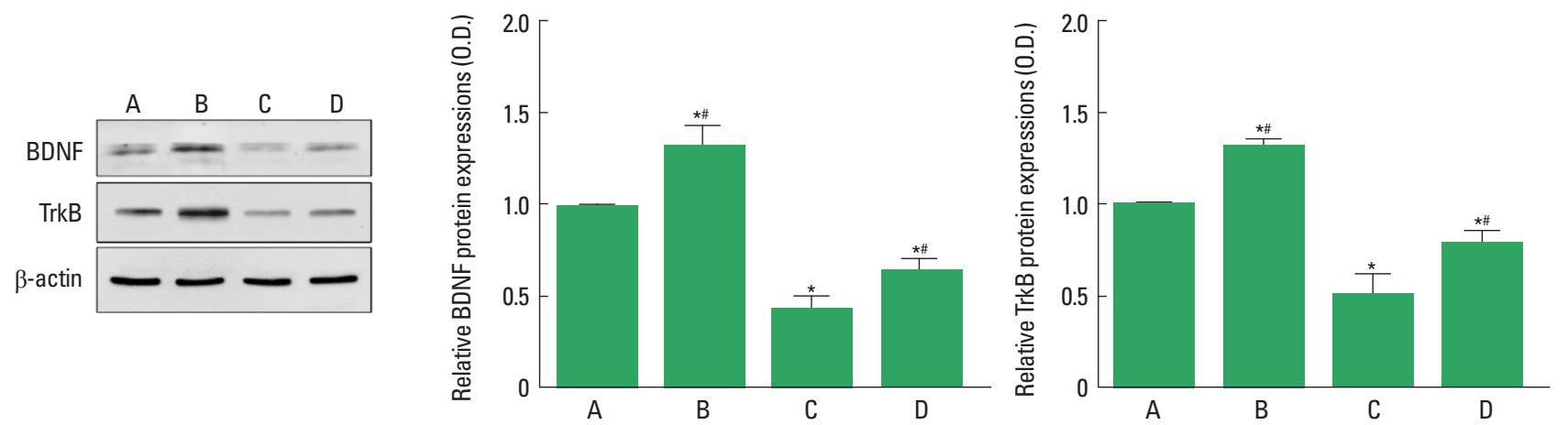

Fig. 2. Effect of paternal treadmill exercise on hippocampal brain-derived neurotrophic factor (BDNF) and tyrosine kinase B receptor (TrkB) expressions in male rat pups. Left panel: Western blot of BDNF and TrkB expressions. Middle \& right panel: BDNF and TrkB expressions in each group. A, paternal nonexercised male pups from normal maternal rats; $B$, paternal exercised male pups from normal maternal rats; $C$, paternal nonexercised male pups from obese maternal rats; $D$, paternal exercised male pups from obese maternal rats. Data are expressed as the mean \pm standard error of the mean. ${ }^{*} P<0.05$ compared to the paternal nonexercised male pups from normal maternal rats. ${ }^{\sharp} P<0.05$ compared to the paternal nonexercised male pups from obese maternal rats.

\section{Effect of paternal treadmill exercise on cell differentiation in male rat pups}

We used DCX labeling to assess cell differentiation in the hippocampus (Fig. 3). Cell differentiation in the hippocampus was decreased in the male rat pups from the obese maternal rats compared to the male rat pups from the normal maternal rats. Paternal exercise increased cell differentiation in the male rat pups from the obese maternal rats.

\section{Effect of paternal treadmill exercise on cell proliferation in male rat pups}

We used BrdU labeling to assess cell proliferation in the hippocampus (Fig. 4). Cell proliferation in the hippocampus was decreased in the male rat pups from the obese maternal rats com- pared to the male rat pups from the normal maternal rats. Paternal exercise increased cell proliferation in the male rat pups from the obese maternal rats.

\section{DISCUSSION}

Maternal nutrients exerted significant effect on the development and maintenance of brain function of offspring (Tozuka et al., 2009a). Maternal high-fat diet provided a hypothalamic leptin resistance in offspring, however, fails to increase the body weight gain until adulthood (Férézou-Viala et al., 2007). C57BL/6 mice received high-fat diet for 6 weeks, and reduced expression of BDNF, disturbed spatial cognitive function, increased lipid peroxidation, and reduced neurogenesis were observed in the off- 

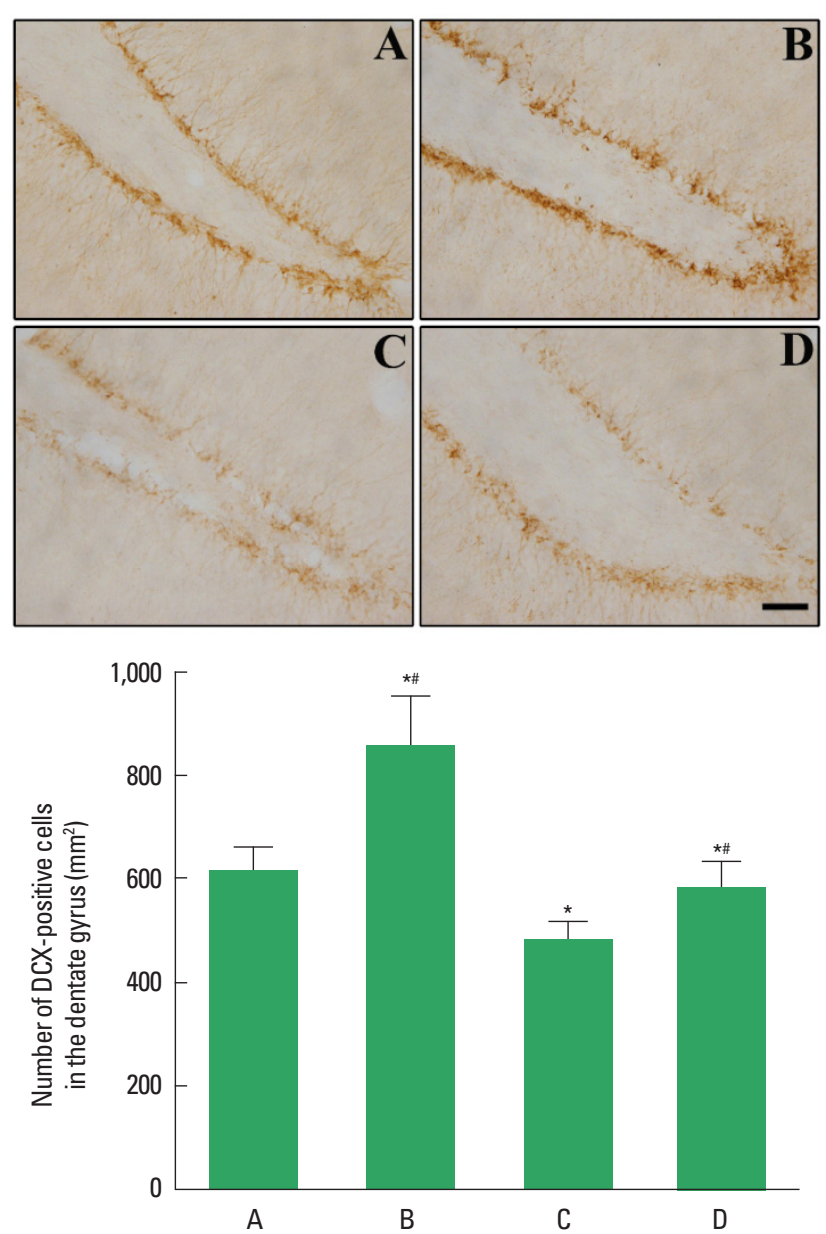

Fig. 3. Effect of paternal treadmill exercise on hippocampal cell differentiation in male rat pups. Upper panel: Photomicrographs of DCX-positive cells. The scale bar represents $100 \mu \mathrm{m}$. Lower panel: The number of DCX-positive cells in each group. A, paternal nonexercised male pups from normal maternal rats; $B$, paternal exercised male pups from normal maternal rats; $C$, paternal nonexercised male pups from obese maternal rats; $D$, paternal exercised male pups from obese maternal rats; $D C X$, doublecortin. Data are expressed as the mean \pm standard error of the mean. ${ }^{*} P<0.05$ compared to the paternal nonexercised male pups from normal maternal rats. ${ }^{\sharp} P<0.05$ compared to the paternal nonexercised male pups from obese maternal rats.

spring born from the obese mother (Tozuka et al., 2009b; Tozuka et al., 2010).

In the present study, obese maternal rats were mad by feeding with high-fat diet for 18 weeks. Spatial learning ability in the Morris water maze task was reduced in the male rat pups born from the obese maternal rats. Expressions of BDNF and TrkB in the hippocampus were suppressed in the male rat pups born from the obese maternal rats. Cell proliferation and differentiation in the hippocampus were reduced in the male rat pups born from the obese maternal rats.
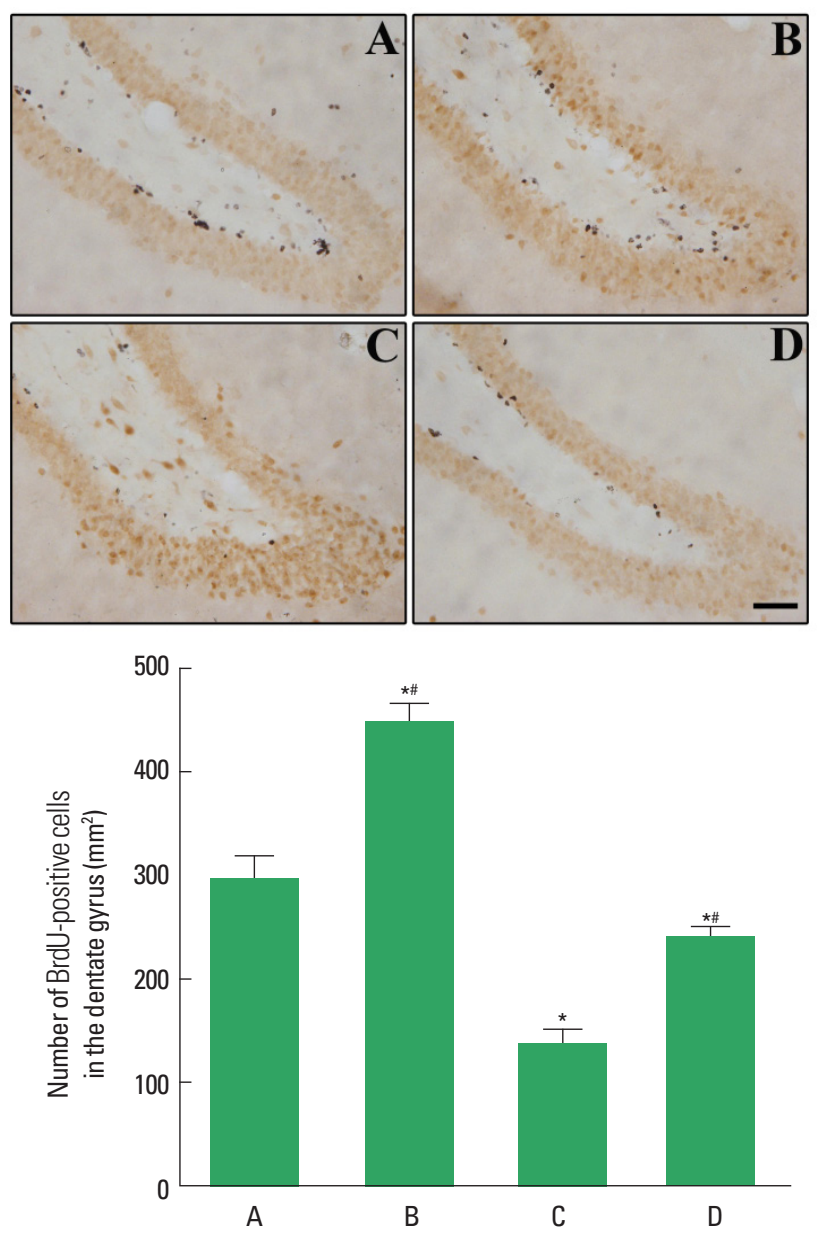

Fig. 4. Effect of paternal treadmill exercise on hippocampal cell proliferation in male rat pups. Upper panel: Photomicrographs of BrdU-positive cells. The scale bar represents $100 \mu \mathrm{m}$. Lower panel: The number of BrdU-positive cells in each group. A, paternal nonexercised male pups from normal maternal rats; $B$, paternal exercised male pups from normal maternal rats; $C$, paternal nonexercised male pups from obese maternal rats; $D$, paternal exercised male pups from obese maternal rats; BrdU, 5-Bromo-2'-deoxyuridine. Data are expressed as the mean \pm standard error of the mean. ${ }^{*} P<0.05$ compared to the paternal nonexercised male pups from normal maternal rats. ${ }^{\sharp} P<0.05$ compared to the paternal nonexercised male pups from obese maternal rats.

Exercise increases neurogenesis in the hippocampus, and exercise is also known to improve hippocampus-dependent learning ability and memory function (Cotman and Berchtold, 2002; Vaynman et al., 2007). The enhancing effect of treadmill exercise on spatial learning ability could be ascribed to the increment of neurogenesis (Kim et al., 2013b).

Paternal obesity increases damage to sperm DNA, as a result, reduced the rate of pregnancy and birth (Bakos et al., 2011). Paternal age and nutrition influence the development of offspring (Curley et al., 2011) and paternal environment also exert impact 
on the brain development of offspring (Mychasiuk et al., 2012). Exercise improved metabolic status and sperm function in obese men (Palmer et al., 2012). Paternal treadmill exercise enhanced hippocampus-dependent spatial learning and memory in the male pups (Yin et al., 2013).

In the present study, paternal treadmill exercise improved spatial learning ability in the male rat pups born from the obese maternal rats. Paternal treadmill exercise increased BDNF and TrkB expressions in the male rat pups born from the obese maternal rats. Cell proliferation and differentiation in the male rat pups born from the obese maternal rats were facilitated by paternal treadmill running. These results suggest that paternal exercise enhanced hippocampal neuroplasticity and consequently improved spatial learning ability in the rat pups born of the obese maternal rats.

Based on the present results, it can be concluded that maternal obesity deteriorates brain function of offspring, in contrast, paternal exercise improves brain function through enhancing neuroplasticity of offspring born of obese mother.

\section{CONFLICT OF INTEREST}

No potential conflict of interest relevant to this article was reported.

\section{ACKNOWLEDGMENTS}

This work was supported by the National Research Foundation of Korea Grant funded by the Korean Government (NRF-2014S1 A5B5A07041321).

\section{REFERENCES}

Akhavan MM, Emami-Abarghoie M, Safari M, Sadighi-Moghaddam B, Vafaei AA, Bandegi AR, Rashidy-Pour A. Serotonergic and noradrenergic lesions suppress the enhancing effect of maternal exercise during pregnancy on learning and memory in rat pups. Neuroscience 2008; 151:1173-1183.

Bakos HW, Henshaw RC, Mitchell M, Lane M. Paternal body mass index is associated with decreased blastocyst development and reduced live birth rates following assisted reproductive technology. Fertil Steril 2011;95:1700-1704.

Bramham CR, Messaoudi E. BDNF function in adult synaptic plasticity: the synaptic consolidation hypothesis. Prog Neurobiol 2005;76:99-125.

Cotman CW, Berchtold NC. Exercise: a behavioral intervention to enhance brain health and plasticity. Trends Neurosci 2002;25:295-301.
Curley JP, Mashoodh R, Champagne FA. Epigenetics and the origins of paternal effects. Horm Behav 2011;59:306-314.

Donovan MH, Yamaguchi M, Eisch AJ. Dynamic expression of TrkB receptor protein on proliferating and maturing cells in the adult mouse dentate gyrus. Hippocampus 2008;18:435-439.

Elias MF, Elias PK, Sullivan LM, Wolf PA, D'Agostino RB. Obesity, diabetes and cognitive deficit: The Framingham Heart Study. Neurobiol Aging 2005;26 Suppl 1:11-16.

Férézou-Viala J, Roy AF, Sérougne C, Gripois D, Parquet M, Bailleux V, Gertler A, Delplanque B, Djiane J, Riottot M, Taouis M. Long-term consequences of maternal high-fat feeding on hypothalamic leptin sensitivity and diet-induced obesity in the offspring. Am J Physiol Regul Integr Comp Physiol 2007;293:R1056-1062.

Fuchs E, Gould E. Mini-review: in vivo neurogenesis in the adult brain: regulation and functional implications. Eur J Neurosci 2000;12:22112214.

Kim H, Lee SH, Kim SS, Yoo JH, Kim CJ. The influence of maternal treadmill running during pregnancy on short-term memory and hippocampal cell survival in rat pups. Int J Dev Neurosci 2007;25:243-249.

Kim SE, Ko IG, Park CY, Shin MS, Kim CJ, Jee YS. Treadmill and wheel exercise alleviate lipopolysaccharide-induced short-term memory impairment by enhancing neuronal maturation in rats. Mol Med Rep 2013a;7:31-36.

Kim TW, Shin MS, Park JK, Shin MA, Lee HH, Lee SJ. Treadmill exercise alleviates prenatal noise stress-induced impairment of spatial learning ability through enhancing hippocampal neurogenesis in rat pups. J Exerc Rehabil 2013b;9:451-456.

Kodomari I, Wada E, Nakamura S, Wada K. Maternal supply of BDNF to mouse fetal brain through the placenta. Neurochem Int 2009;54:95-98.

Krawetz SA. Paternal contribution: new insights and future challenges. Nat Rev Genet 2005;6:633-642.

Lehner B, Sandner B, Marschallinger J, Lehner C, Furtner T, Couillard-Despres S, Rivera FJ, Brockhoff G, Bauer HC, Weidner N, Aigner L. The dark side of BrdU in neural stem cell biology: detrimental effects on cell cycle, differentiation and survival. Cell Tissue Res 2011; 345:313-328.

Lie DC, Song H, Colamarino SA, Ming GL, Gage FH. Neurogenesis in the adult brain: new strategies for central nervous system diseases. Annu Rev Pharmacol Toxicol 2004;44:399-421.

Mychasiuk R, Zahir S, Schmold N, Ilnytskyy S, Kovalchuk O, Gibb R. Parental enrichment and offspring development: modifications to brain, behavior and the epigenome. Behav Brain Res 2012;228:294-298.

Palmer NO, Bakos HW, Owens JA, Setchell BP, Lane M. Diet and exercise in an obese mouse fed a high-fat diet improve metabolic health and reverse perturbed sperm function. Am J Physiol Endocrinol Metab 
2012;302:E768-780.

Raiten DJ, Kalhan SC, Hay WW Jr. Maternal nutrition and optimal infant feeding practices: executive summary. Am J Clin Nutr 2007;85:577S5835 .

Tozuka Y, Kumon M, Wada E, Onodera M, Mochizuki H, Wada K. Maternal obesity impairs hippocampal BDNF production and spatial learning performance in young mouse offspring. Neurochem Int 2010;57:235-247.

Tozuka Y, Wada E, Wada K. "Bio-communication" between mother and offspring: lessons from animals and new perspectives for brain science. J Pharmacol Sci 2009a;110:127-132.

Tozuka Y, Wada E, Wada K. Diet-induced obesity in female mice leads to peroxidized lipid accumulations and impairment of hippocampal neurogenesis during the early life of their offspring. FASEB J 2009b;23: 1920-1934.

Vaynman S, Ying Z, Gomez-Pinilla F. The select action of hippocampal calcium calmodulin protein kinase II in mediating exercise-enhanced cognitive function. Neuroscience 2007;144:825-833.

Wolf PA, Beiser A, Elias MF, Au R, Vasan RS, Seshadri S. Relation of obesity to cognitive function: importance of central obesity and synergistic influence of concomitant hypertension. The Framingham Heart Study. Curr Alzheimer Res 2007;4:111-116.

Yin MM, Wang W, Sun J, Liu S, Liu XL, Niu YM, Yuan HR, Yang FY, Fu L. Paternal treadmill exercise enhances spatial learning and memory related to hippocampus among male offspring. Behav Brain Res 2013; 253:297-304. 